\title{
How do apes ape?
}

\author{
ANDREW WHITEN, VICTORIA HORNER, \\ CARLA A. LITCHFIELD, and SARAH MARSHALL-PESCINI \\ University of St. Andrews, St. Andrews, Scotland
}

\begin{abstract}
In the wake of telling critiques of the foundations on which earlier conclusions were based, the last 15 years have witnessed a renaissance in the study of social learning in apes. As a result, we are able to review 31 experimental studies from this period in which social learning in chimpanzees, gorillas, and orangutans has been investigated. The principal question framed at the beginning of this era, Do apes ape? has been answered in the affirmative, at least in certain conditions. The more interesting question now is, thus, How do apes ape? Answering this question has engendered richer taxonomies of the range of social-learning processes at work and new methodologies to uncover them. Together, these studies suggest that apes ape by employing a portfolio of alternative social-learning processes in flexibly adaptive ways, in conjunction with nonsocial learning. We conclude by sketching the kind of decision tree that appears to underlie the deployment of these alternatives.
\end{abstract}

The expression to ape suggests that the three genera most closely related to ourselves ought to offer particularly rich pickings in the investigation of animal social learning. There are several reasons why the generous space accorded to the great apes, so small an animal taxon by comparison with others reviewed in this issue, might be justified.

The first concerns the primary functional significance of social learning, which for primates has long been hypothesized to lie in the acquisition of adaptive traditions, such as antipredator behavior and, particularly, skilled or clever foraging methods (Imanishi, 1957; Nishida, 1987; Passingham, 1982). The discovery of a variety of forms of chimpanzee tool use, together with the close observation of these by juveniles and the patterns of acquisition associated with them, was soon interpreted as indicating the existence of socially learned traditions, or cultures (Goodall, 1968, 1973). As other long-term studies were carried out across Africa, collation of the published results produced a growing list of putative cultural variations (McGrew, 1992). Most recently, recognizing that a reliance on published data might yield only a partial picture of the existing variations, research site leaders have pooled their long-term records in a two-phase procedure designed to establish a more definitive picture (Whiten et al., 1999, 2001). By first generating a set of hypothesized variations and then sieving these through a systematic coding procedure at each of nine sites across

For comments on earlier versions of this manuscript, we are grateful to Christine Caldwell, Tara Stoinski, and particularly for her incisive editorial advice, Celia Heyes. We thank Jeff Galef for drawing our attention to Lloyd Morgan's early discussion of the issue of emulation. Correspondence concerning this article should be addressed to A. Whiten, Centre for Social Learning and Cognitive Evolution and Scottish Primate Research Group, School of Psychology, University of St. Andrews, St. Andrews, Fife KY16 9JU, Scotland (e-mail: a.whiten@st-and.ac.uk).
Africa, researchers have identified no fewer than 39 different behavior patterns as likely cultural variants, occurring commonly in at least one community, yet absent in another, with no apparent environmental or genetic explanations for this patterning. This procedure has more recently been applied to orangutans, identifying 19 cultural variants, plus five more tentative candidates (van Schaik et al., 2003). Van Schaik et al. additionally tested two predictions based on the hypothesis that the variants are transmitted by social learning. These predictions were supported for both chimpanzees and orangutans: Geographically closer communities share a greater proportion of variants, and communities in which individuals spend more time in close proximity have more extended repertoires of variants.

In these studies, a substantial contrast with studies of traditions among other species, which typically identify only one or a small handful of variations, is highlighted. This suggests that in the evolutionary spiraling of cultural complexity that has apparently occurred, apes have been subject to selective pressures that have sculpted the strength and nature of mechanisms for social learning in special ways: a case of gene-culture coevolution(Laland, Odling-Smee, \& Feldman, 2000). Observation of apes' natural behavior was essential to gain this functional perspective but, unfortunately, offers little prospect of further untangling the many different learning processes that could be operative. For this purpose, experimentation offers distinctive power, and given our space limits here, the results of such studies are the principal focus of the present review. Which social-learning processes are associated with the phenomena of traditions and cultures remains a major, yet unresolved issue in animal learning (Galef, 1992, 2004; Heyes, 1993).

A second reason why social learning among great apes begs attention derives directly from their being our closest living relatives. Identifying aspects of ape social-learning 
capacities shared with humans permits inferences about these abilities in our shared ancestors of 5-6 million years ago. Such comparisons should equally sharpen our understanding of just where the critical changes in social learning and culture occurred in our evolutionary past (Whiten, Horner, \& Marshall-Pescini, 2003).

A third set of reasons to value ape research involves linkages with other disciplines. For example, both theoretical and methodological aspects of research on ape social learning have recently shaped related work in child development (Want \& Harris, 2002; Whiten, 2002c). Other links are developing with artificial intelligence and robotics (Dautenhahn \& Nehaniv, 2002), as well as with neurophysiology and neuropsychology (Hurley \& Chater, in press; Meltzoff \& Prinz, 2002). Animal social learning lies at the intersection of a number of such exciting developments, with ape research playing a significant role, for the reasons outlined above.

\section{THE RECENT HISTORY OF RESEARCH ON APE SOCIAL LEARNING}

The last 15 years have seen an upsurge in systematic studies of social learning in apes, the conclusions of which we will review below. We should begin, however, by setting this recent work in its wider historical context.

Anecdotal reports of imitation by apes stretch back for centuries, and more systematic studies of social learning were pursued all through the last century (Mitchell, 1999; Tomasello \& Call, 1997; Whiten \& Ham, 1992). The 20th century's efforts included formal experiments, ethological studies in the wild, and observations of captive apes, some of the latter being reared in human families, facilitating detailed comparison with the behavior of human children in similar contexts. By the 1970s, a common conclusion was that apes ape. Influentially, K. J. and C. Hayes's (C. Hayes, 1951; K. J. Hayes \& C. Hayes, 1952) studies of the home-reared chimpanzee Vicki included graphic accounts of the way she copied such everyday human activities as brushing one's teeth, plus the results of an experimental battery of tests in which she was reported to respond convincingly, after training, to the request to "do this," even for relatively novel actions. Another influential strand was the discovery of what looked like apprenticeship to skilled tool use in the wild (Goodall, 1973). Such diverse sources appeared to converge on a conclusion fairly routinely expressed in textbooks, that apes possess a functionally important capacity to imitate; indeed, this was sometimes interpreted as just a fuller expression of a capacity probably quite widespread in primates (e.g., MacFarland, 1984; Passingham, 1982).

Beginning in the late 1980 s, this picture was severely challenged by a string of publications. One of the first described an experiment that used conspecific models, rather than human demonstrators (Tomasello, Davis-DaSilva, Camak, \& Bard, 1987). Instead of a chimpanzee such as Vicki, influenced by rich interactions with human foster parents who were also her models, group-living captive chimpanzees were tested and showed only limited powers of social learning. Observer chimpanzees who watched an expert conspecific using a stick to rake food from an out-of-reach platform were more likely to become rakers than were control subjects who did not see a model, but they failed to copy the particular two-step technique the model had used to retrieve trapped food.

Such a result obtained in a chimpanzee-chimpanzee test raised the disturbing possibility that the average chimpanzee might not really deserve its reputation for imitation after all. On the heels of this finding came a number of influential critiques of earlier social-learning studies. Largely on methodological grounds, these publications questioned widely held suppositions about the propensity for imitation in primates and other animals (Visalberghi \& Fragaszy, 1990; Whiten \& Ham, 1992), including apes (Galef, 1988, 1990; Heyes, 1993; Tomasello, 1990).

This critical onslaught offered a challenge to received wisdoms and stimulated a raft of new studies, displaying greater sophistication in both theory and method. Among these, observational and field studies have played a crucial role, as was noted at the outset of this article. However, it is difficult through pure observation to dissect the causal processes underlying social learning. Accordingly, the focus of the present review is the experimental studies published since Tomasello et al.'s influential 1987 report. We will review 31 of these, a number that represents a renaissance of research in this area, contrasting with the mere 4 or so such studies that Tomasello and Call (1997) listed for the 15 years before the "watershed" year of 1987. There is not the space here to describe all of the studies in detail. Instead, we shall attempt to draw out emerging general conclusions, illustrating them with appropriate examples.

\section{DO APES APE?}

The developments summarized above meant that the urgent question in the 1990s was whether apes do or do not, after all, ape- a question that most researchers, like Tomasello (1996), took to be synonymous with asking Do they imitate? (imitation will, in turn, be defined and dissected in the next section). A common conclusion about social learning among primates was that monkeys, despite a century's efforts, had not been shown to ape in this sense, whereas the evidence for apes themselves was more ambiguous (Shettleworth, 1998; Whiten \& Ham, 1992). Apes had generated a curious mixture of negative and positive findings, some of the latter alluringly vivid, even if anecdotal. Tomasello (1996) portrayed imitation as an inherently sophisticated cognitive achievement, appearing clearly only in humans, the prime question being whether our closest animal relatives might just achieve the same mental heights.

Largely through the use of more robust experiments, the pendulum has since swung back from such extremes of doubt over whether nonhuman animals imitate. In the Appendix, we list the accumulated evidence for apes, including a column that summarizes the authors' key con- 
clusions. As is evident, imitation figures prominently; indeed, for chimpanzees and orangutans, it is the most common conclusion. The existence of imitation in apes is now accepted in relation to at least some of these studies by the more skeptical of the earlier critics (Galef, in press; Heyes, 2001). Accordingly, we can move forward to the more difficult, but interesting, question, How do apes ape?

Taking this step now appears not so radical, because evidence for imitation has also recently accumulated for other, very different species. Of two studies in the refereed literature allowed to include true imitation in their titles, one concerned marmosets (Voelkl \& Huber, 2000) and the other pigeons (Zentall, Sutton, \& Sherburne, 1996). Several other recent studies have demonstrated imitation in small birds (see Zentall, 2004), whose brains are tiny, as compared with those of the great apes. Of course, there may be important differences between what we can broadly call imitation in apes and that in species so different from them, and we shall consider these further below.

\section{How Apes Ape: Imitation and Alternative Social-Learning Processes}

Essential preliminaries to our analysis of how apes ape include clarifying what we intend the verb ape to connote and what, in the present state of knowledge, we can hope to include in the how.

The terminology and underlying conceptual framework of social-learning research has become infamous for its complexities and confusions. Disputes about whether apes (and other species) imitate have often focused on what is deemed an acceptable definition of imitation, as much as on what reliable data a study has gathered. However, we believe that there will be a net benefit if, from within these debates, we can now distill a more refined dissection of alternative processes of social learning.

Perhaps the most influential new distinction arose from the 1987 article by Tomasello et al., already highlighted. Finding that chimpanzees learned something about tool use that nonobserving control subjects did not, the authors concluded that social learning was involved. However this was said not to involve imitation, because the subjects did not copy the particular two-stage technique the model had used to gain food trapped at the edge of the platform. Even so, the authors rejected the hypothesis that the learning involved stimulus enhancement-attention being drawn to relevant objects-because the control subjects manipulated the stick as much as the observers and were motivated to attempt to obtain the food. Instead, Tomasello et al. (1987) concluded that the experimental subjects had learned by observation something about the stickspecifically, its function as a tool. Tomasello (1990) later labeled this emulation.

Since 1990, the meaning of emulation has diversified, in sometimes confusing ways. Nevertheless, we suggest that within this varied usage, some important distinctions are worth making more explicit and operationalizing, a task we will pursue in the next section. Moreover, we interpret some of the varieties of what has been called emulation as overlapping with imitation in important ways, rather than as offering a neat dichotomy. Accordingly, we will consider how apes ape in both a broad and a narrower sense. The narrower sense may be equated with imitation, although imitation may itself be defined in the literature either in relatively restricted ways (e.g., limited to only bodily imitation or to novel actions or requiring the recognition of the model's intent) or, instead, more liberally, not requiring these constraints. Assessing how apes ape in a yet broader sense requires us to analyze how imitation may operate as part of a portfolio of other social-learning processes and, indeed, may overlap with some of these. In doing this, we intentionally echo the approach taken by Want and Harris (2002), who considered a panoply of social-learning processes, including stimulus enhancement, emulation, and imitation, in answering the question, How do children ape?

The classification of social learning we shall apply here is depicted in Figure 1. It represents an updated version of part of the scheme presented by Whiten and Ham (1992), considerably revised in the light of theoretical distinctions made in the intervening period. In asking how apes ape, we appraise the growing corpus of empirical evidence that suggests how apes deploy the options suggested by Figure 1. We are not proposing to answer our question at the level of what psychological or neurobiological processes constitute the underlying mechanisms of imitation. The discovery of mirror neurons in monkeys has fueled recent theorizing about such mechanisms (Byrne, 2000; Heyes, 2002; Rizzolatti, Fadiga, Fogassi, \& Gallese, 2002; Whiten, 2000), but since no such work has been done on great apes, we think the level at which we here tackle our overarching question is the appropriate and productive one at the present time.

Given this approach, it is important to recognize that although our intention is that the categories of learning discriminated in Figure 1 be conceptually and experimentally separable, it is important to recognize that more than one might be in operation in any single episode of social learning; they are not all mutually exclusive in this respect. Through the main sections to follow, we will work from the top of Figure 1 down, explaining the distinctions between our categories with a focus on different senses of emulation that we think beg particular attention. We will begin by comparing one of these with imitation. Key findings for apes are noted in relation to each distinction in turn.

\section{How Do Apes Ape?}

1a. Imitation and object movement reenactment: Distinctions and overlaps. Following Whiten and Ham (1992), we define imitation as a process whereby one individual copies some part of the form of an action from another. By contrast, according to Tomasello (1998), in emulation learning, animals are "learning about the environment, not about behaviour" (p. 704). Where exactly is the critical boundary between the two? The essential distinction implied is that an imitator is learning about 


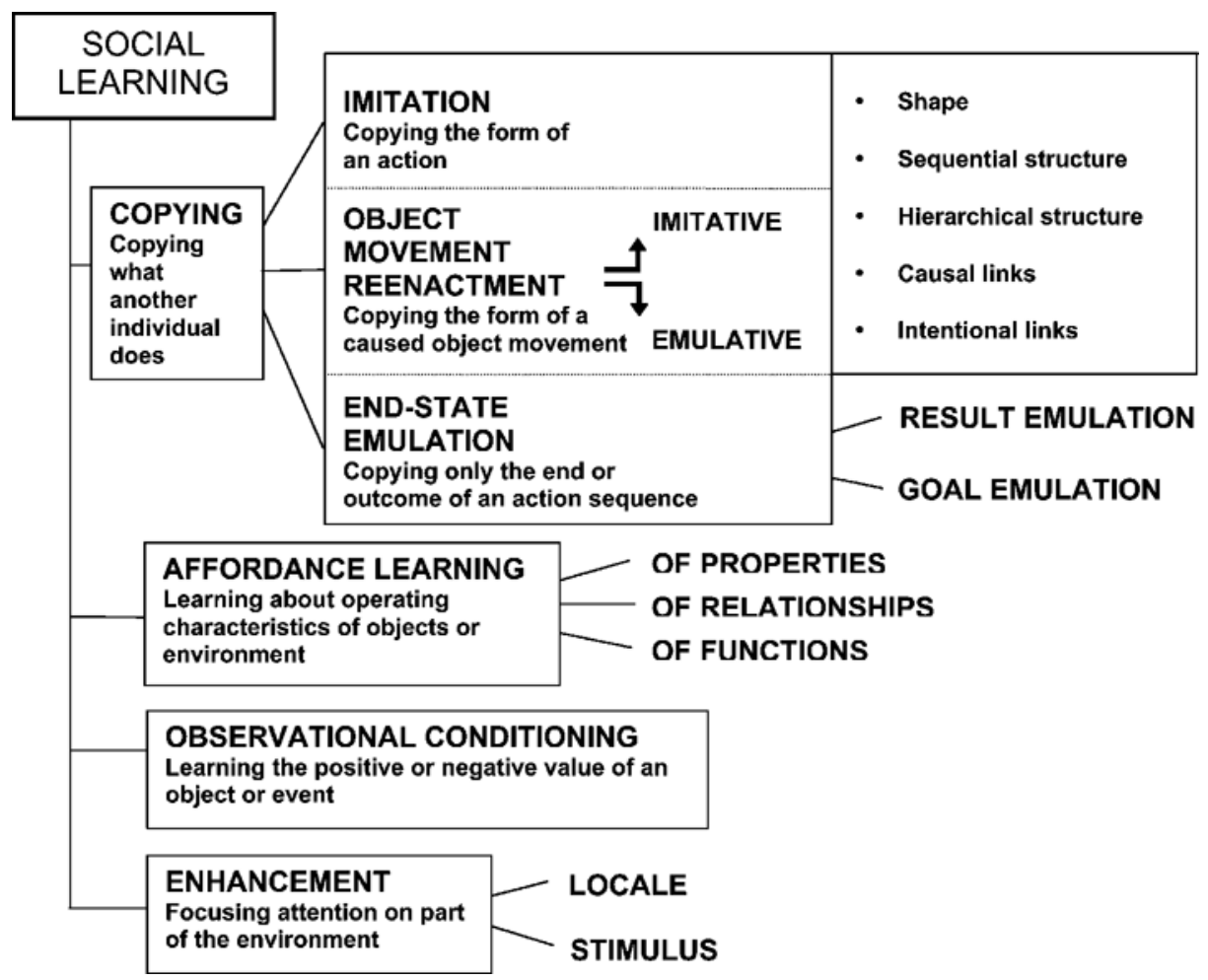

Figure 1. A taxonomy of social-learning processes. This scheme represents a revision of that offered in Whiten and Ham (1992) that takes into account theoretical and empirical progress in the intervening period. Note that the categories presented here omit those Whiten and Ham classed as only social influence, such as social facilitation. In the present scheme, different kinds of sociallearning processes are discriminated, labeled, and partially defined (for further specification, see the text). Categories are arranged so that there is generally most potential for high-fidelity copying at the top (imitation), descending to enhancement at the bottom. Affordance learning may, in principle, be used to generate actions that vary in their degree of match to actions of the individual(s) observed. To the right of the "Imitation" and "Object Movement Reenactment" (OMR) categories are listed a number of different aspects in relation to which matching to a model may occur-for example, hierarchical structure might be copied in relation to either imitation of bodily movements or OMR. The same is true for the spatiotemporal shape of actions, their sequential patterning, and causal and intentional aspects of the model's actions. Note that Whiten and Ham labeled all emulation as goal emulation, whereas here, goal emulation is only a subcategory of emulation, for which a tighter specification is explained in the text.

events bounded within the skin of the model, whereas an emulator is learning about events outside the models' skin (where the environment begins). Such a distinction may reflect significant differences in the underlying information-processing mechanisms, although this remains to be established. One way to investigate the distinction is the ghost control (Heyes, Jaldow, Nokes, \& Dawson, 1994), in which, through the experimenter's ingenuity, the observer animal sees the environmental changes normally caused by the model, but without actually seeing what the model does to make them happen.

However, emulation defined in this way could well involve information processing that shares fundamental similarities with the imitation of bodily movements. Consider cases in which an observer reproduces the movements of objects caused by a model, a category that Custance, Whiten, and Fredman (1999) have described as “object movement re-enactment" (OMR; see Figure 1).
Here, the observer is essentially copying what the object does (or to put it another way, what the model does with the object), in a way that appears analogous to copying what body parts do. Accordingly, in Figure 1, imitation and OMR are classified together as copying processes. To illustrate the point with an example, if A copies the trajectory of a hammer swung by $\mathrm{B}$, this may draw on imitative processes not so different from those involved when A copies the way B hammers with a fist (Whiten \& Custance, 1996). Given that a tool, such as a hammer, can come to be effectively an extension of the holder's limb, sharing characteristics of the way in which the hand functions as a natural tool of the arm (Whiten, 2000), the body-environment boundary may turn out not to be such a critical one at which to expect a fundamental difference in social-learning mechanism. For example, the results of the study of Tomasello et al. (1987) may be explicable as a case of low-fidelity OMR in 
which observers copied the actions of the stick at a relatively crude level (describable as raking), rather than at the detailed level of the two-step method the model had used in certain contexts.

One respect in which bodily imitation might differ fundamentally from OMR concerns cases in which the copier cannot see its own actions, such as picking ones teeth with one's fingers. Copying such perceptually opaque actions can be expected to impose distinctive information-processing requirements (Heyes, 2002). However, object movements may also be opaque, as in using a brush to clean one's teeth or applying lipstick.

One could even extend the above logic to include OMR within a category labeled imitation (which would then include bodily imitation and object movement imitation; Stoinski, Wrate, Ure, \& Whiten, 2001; Whiten, 2000). However, it remains an open empirical question as to how similar to bodily imitation copying of the various kinds of object movements that an animal can cause is. As was indicated above, some may be opaque, and others not. Some may be intimately interwoven with bodily actions, such as hammering, but others may be much more distal, such as objects being moved by the end of a tool. Accordingly, in Figure 1, we refrain from simply assimilating OMR to imitation but indicate the possibility that cases of OMR may vary in the extent to which the information processing involved shares characteristics with imitation. This is a more messy approach but expresses potential differences and similarities that beg empirical investigation, in contrast to a too easily accepted imitation/emulation dichotomy.

1b. Imitation and OMR in apes. Beginning with imitation, we note two importantly different questions: $\mathrm{Can}$ apes imitate? versus Do apes imitate? Different methodological approaches have differential power in tackling these. In relation to the first, the technique described as do-as-I-do offers the opportunity, once a subject has been trained to the basic idea of attempting to copy what a model does on command, of exploring quite fully just what they can and cannot copy. It has been successfully implemented with both chimpanzees (Custance, Whiten, \& Bard, 1995; K. J. Hayes \& C. Hayes, 1952; MyowaYamakoshi \& Matsuzawa, 1999; Tomasello, SavageRumbaugh, \& Kruger, 1993) and orangutans (Call, 2001; Miles, Mitchell, \& Harper, 1996) with quite consistent findings that cover not only bodily imitation, but also OMR (in the Appendix, for each genus, do-as-I-do studies are the first listed).

A number of significant conclusions have flowed from this work. (1) The do-as-I-do method indicates that some apes, at least, can imitate, because coders blind to what subjects saw can identify matches at statistically significant levels. (2) Imitation applies to a range of actions that includes facial expressions, unimanual and bimanual gestures, and bodily postures (e.g., Custance et al., 1995), as well as the copying of object manipulations (OMR; e.g., Tomasello, Savage-Rumbaugh, \& Kruger, 1993). (3) Sometimes, subjects respond initially with actions already known, which only approximate what the model did, and then gradually "home in" on the model's action. (4) Touching body parts out of sight (an example of Heyes's, 2002, perceptually opaque actions) may be achieved as accurately as those in sight. (5) As compared with children, who may show recognizable matching on all of the actions in the battery used, fidelity is typically low overall. (6) Successful imitation can be deferred (48 $\mathrm{h}$ in the study of Tomasello, Savage-Rumbaugh, \& Kruger, 1993). Applying the same approach to OMR, copying the movements of one object in relation to another was found to be easier than copying the movements of a single object, including applying it to oneself (Myowa-Yamakoshi \& Matsuzawa, 1999).

The alternative question-Do apes imitate?-is more often appropriate in experiments in which the observer is allowed to watch a model and investigation focuses on what the observer subsequently does freely. The question becomes What does the observer learn through observation? with imitation being merely one of the possibilities. This approach commonly uses functional tasks, such as gaining food through tool use or some other kind of manipulation. These tasks are often designed to be relevant to the question of whether apes are likely to acquire their traditions in the wild through imitation or other learning processes, an example of which is our use of artificial fruits (Whiten, 2002a). Such studies are grouped, for each genus, in the lower parts of the Appendix.

There has been much disagreement about how apes ape-what information they are processing — when faced with observation of such tasks. As we have seen, Tomasello et al. (1987) interpreted their results as showing that chimpanzees emulate, rather than imitate. Nagell, Olguin, and Tomasello (1993) and Call and Tomasello (1995) concurred in this conclusion. In contrast, using the opening of a complex artificial fruit as the task, instead of tool use, Whiten, Custance, Gomez, Teixidor, and Bard (1996; Whiten, 1998b) and Stoinski et al. (2001) obtained results for chimpanzees and gorillas, respectively, that they interpreted as evidence for both imitation and emulation. In these studies, chimpanzees copied by either poking bolts out of holes or, instead, pulling and twisting them out, according to whichever of two alternative models they had seen. Similarly, gorillas matched their models in either pulling a pin straight out of its hole or removing it by twisting.

As Tomasello (1996) noted, these results might still reflect emulation (in the sense of OMR) if subjects were not replicating bodily movements but, instead, had learned only about the objects themselves. These alternatives may often be difficult to disambiguate in object manipulation tasks. However, Stoinski et al. (2001) found that unlike the chimpanzees studied by Whiten et al. (1996), gorillas did not copy poking versus twisting of the bolts when faced with a similar "fruit." They did, however, replicate the way the bolts moved, either away from the manipulator or (in the alternative experimental condition) toward them. This is consistent with the gorillas' focusing on what the objects themselves "do" and evidencing OMR, rather than bodily imitation. 
However, we interpret the twisting actions observed as bodily imitation, because the hands of the model obscured the object manipulated (Custance, 1998). Moreover, in more recent unpublished studies with wild-born chimpanzees in an African sanctuary, we have recorded multiple poking of the bolts back and forth by those subjects who saw the model poke, consistent with imitation, but not with OMR.

The artificial fruit studies have thus been interpreted as evidencing imitation in two different senses. One, adopting the more restricted definition of imitation as bodily rather than object centered, would lead to the conclusion that there is some evidence of this (although relatively little in comparison with children, which will be discussed further below). Alternatively, by assimilating OMR to a broader sense of imitation as outlined earlier (Stoinski et al., 2001), more of the copying recorded would be seen as indicating imitative learning.

The overall picture of imitation from our own studies is thus one in which both bodily and object movement copying occurs. However, the former typically plays a relatively minor role in functional tasks that involve object manipulation. This may sometimes reflect limitations in imitative capacity, but on other occasions, it may reflect adaptive choices, where it can be more efficient to replicate object movement through actions that do not match those of the model. That apes' primary focus of attention is on what the objects or environmental features manipulated are doing is a conclusion about which we begin to see some real consensus between different research groups. As has been noted, this was apparent in our own work with artificial fruits, but it has also been emphasized with regard to a finding in Myowa-Yamakoshi and Matsuzawa (1999) that chimpanzees found it easiest to imitate object-object actions and, of course, in the work of Tomasello and colleagues, reviewed above (and see the Appendix), which led them to highlight emulation as another high-level mode of social learning, contrasting with imitation.

2a. Emulation of results and goals: The essential distinctions. A formulation of emulation different from OMR is that "the observer is attending to the end result" (Tomasello, 1998, p. 704). This was clear in Wood's (1989) original introduction of the term, stating that "children attempt to impersonate others by imitating their actions but also try to emulate them by achieving similar ends or objectives" (p. 71); more specifically, emulation includes "instances where children achieve common goals to those modeled, but do so by using idiosyncratic means that were never observed" (p. 72). This contrasts starkly with imitation, for in emulation as defined by Wood, precisely what the observer is not doing is copying the model's approach to the problem. This is why we do not include our corresponding category of end-state emulation under either imitation or OMR.

Nevertheless, the implication is that the observer is copying something - achieving the end result they see the model achieve. Accordingly, we class end-state emulation, imitation, and OMR under the superordinate cat- egory of copying (Figure 1). In this we follow Miklosi (1999), who suggested that the term copying might be an appropriate one for both imitation and some of the phenomena referred to as emulation. We can also appeal here to the great pioneer of social-learning research, Lloyd-Morgan (1896). Anticipating discussion of emulation by a century, Lloyd-Morgan drew out a distinction between reproducing another's actions versus their results (i.e., emulation), noting, however, that "both are commonly called imitation," yet also "both are commonly called copying" (pp. 172-173). Agreeing with Lloyd Morgan, we suggest that it will help to minimize confusion if we keep scientific usage sensibly in line with the terms we borrow from everyday usage, even as we formalize and define them for clarity's sake.

Under end-state emulation, we include emulation of results and goals. This reflects Wood's (1989) reference, quoted above, to emulation of "ends," "objectives," and "goals." Similarly, Tomasello (1990) referred to an emulator's "focus on the demonstrator's goal" (p. 283) and, elsewhere, to "attending to the end result" (1998, p. 704). Both Wood and Tomasello tended to use these terms (result and goal) almost interchangeably to delineate emulation, but there is surely a significant distinction between an emulator who seeks only to re-create an observable result of the model's actions and an emulator who regards the model as an organism with goals and seeks to reproduce what it infers to be the model's goal on a particular occasion. Call and Carpenter (2002) likewise emphasized this distinction in proposing a threeway taxonomy of the information acquired in social learning, referring to actions, results, and goals.

Of course, a result of a model's action can also be its goal; the question is whether the emulator is interpreting the end-state as a goal or, otherwise, just as an observable outcome of interest. Also, where an observer infers a model's goal and later attempts to achieve it, even though the model had failed, we must be dealing with the copying of goals, rather than of results. Interestingly, Meltzoff (1995) described such a result for human infants. He described this as "re-enactment of intentional actions," but this apparent allusion to an imitative process has been challenged through a study by Huang, Heyes, and Charman (2002) that implicated emulation.

2b. Result emulation and goal emulation in apes. Earlier, we suggested that the results in Tomasello et al. (1987) could be a case of OMR. They could alternatively represent result emulation, if all that the observers were learning was that an end-state was possible-" getting the food raked in." In principle, this could be discriminated by allowing observers to see only the end-resultperhaps an image of only the final configuration, with the food and the rake drawn back in reach. Since OMR, by contrast, could involve copying a whole sequence of stick movements, such movements would all need to be seen. Huang et al. (2002) came close to the required experiment with young children, in preventing them from seeing the central actions normally observable in a model's performance. However, in this study, the subjects 
were allowed to see the starting state as well as the endstate. Such experiences were as powerful as having seen a model's behavioral attempts in causing children to achieve the same ends.

We should also note that Wood (1989) described emulation as achieving "common goals to those modeled ... by using idiosyncratic means that were never observed" (p. 71). If we include the latter in defining endstate emulation, the results in Tomasello et al. (1987) do not, in fact, fit very well, insofar as the chimpanzees did not use alternative means to replicate the result achieved by the model when food was in the trapped position; instead, they failed at the task. Crude OMR thus seems a more apt interpretation.

In our studies with artificial fruits, we have repeatedly found that great apes can, and often do, attain the endstates achieved by the model, preferentially using their own methods where these are effective. Thus, in chimpanzee experiments (e.g., Whiten et al., 1996), subjects that had witnessed a model turn a handle to release a lid quickly pulled the handle out instead (the method, in fact, witnessed by the other experimental group). Clearly this is not OMR, by definition; nor does it suggest imitation, for the actions are different. That strategies were not just what subjects might do without the benefit of a model is indicated by our study of gorillas, which included nonobserving subjects. These control animals simply did not interact with the handle part of the fruit (Stoinski et al., 2001). The directness of experimental subjects' actions on the handle leads us to favor end-result emulation over stimulus enhancement as an explanation, although further control studies will be necessary to clarify this.

Perhaps the best finding illustrating end-result emulation comes from an experiment that incorporated social learning, even though this was not its prime focus (Toth, Schick, Savage-Rumbaugh, Sevcik, \& Rumbaugh, 1993). The bonobo Kanzi, having observed a human striking stone flakes and using them to cut a cord to release food, began to make flakes in a similar way, hitting one stone on another. Having made only relatively inefficient flakes, he then switched to a new technique that had not been demonstrated: smashing a large stone on the ground. Later, he began to throw one stone onto another. Both of these creative variants appear to be just the kind of response originally defined as emulation by Wood (1989).

Goal emulation is more difficult than result emulation to identify in practice. Attempting to do so, MyowaYamakoshi and Matsuzawa (2000) studied what chimpanzees learned from watching a human model trying (and sometimes failing) to open a puzzle box. The model either was successful with one technique (using a tool or only the hand) and then unsuccessful with the alternative technique or was first unsuccessful with one and then successful with the other. After the first demonstration, the subjects tended to copy whichever of the approaches they had seen. However, they copied the second demonstration only if it was successful. The authors interpret their results as showing that "the chimpanzees understood something about the intention of the demon- strator in the failure phase" (p. 388). Their logic is that copying in the first attempt-but-fail case implied recognition of what was intended, rather than a simpler process such as stimulus enhancement, because if the latter had been operative the subjects would have copied attempt-but-fail if this occurred in the later trial. However, an alternative explanation is that in the first trial, the subject used the best information available, a failed attempt in this case; when the failed attempt instead came in the second trial, this was not copied, because the subject now knew about a successful technique. MyowaYamakoshi and Matsuzawa's (2000) approach is, nevertheless, an ingenious one that merits further refinement to tackle this critical issue.

3a.Affordance learning: The essential distinctions. Emulation has also been described as learning the affordances of objects, a term borrowed from perception theory and first used in delineating emulation by Tomasello, Kruger, and Ratner (1993). Following Byrne (1998) and Custance et al. (1999), we use the term affordance learning to cover this idea in Figure 1. As Byrne (1998) noted, affordances could include properties of objects (e.g., stone-hammer hardness), relations between objects (kernels are enmeshed in nut-cases), and functions (a stick can act as a rake; Figure 1). Unlike the senses of emulation described in earlier sections, affordance learning does not necessarily invoke a copying process. To the contrary, having learned about the affordances of various objects, an individual may be able to exploit this knowledge not only in the task observed, but in others too. Want and Harris (2002) suggested that the latter possibility could be one way to discriminate affordance learning from the rest; its consequences ought to be reflected in measurable abilities to apply the factual knowledge obtained to comparable but different tasks.

An alternative approach was adopted in a different article by Want and Harris (2001), showing that young children avoided copying a particular segment of a model's actions that was unproductive in arriving at a final desired outcome. The authors interpreted this as the children's learning, instead, about critical affordances in the task (that poking a stick into the wrong end of a tube caused the contents to fall into a trap) and using this information in their task solution. Thus, learning from a model what to ignore might be another useful focus in the investigation of affordance learning.

3b. Affordance learning in apes. Horner and Whiten (2004) have recently completed a study that echoes the logic of Want and Harris (2001) in pursuing the hypothesis that, in certain contexts, chimpanzees will make adaptive choices either to imitate what a model does or to more selectively ignore components that can be judged unproductive in their affordances. In one condition in this study, subjects witnessed a human model who, among other actions, poked a stick-tool into the top of an opaque box and then removed it, finally inserting it into another hole at the front to retrieve a food reward. In an alternative condition, subjects saw exactly the same thing, but the box was transparent, so that when the tool was in- 
serted into the top, an observer could see that it unproductively struck a false floor. As was predicted, the young chimpanzees who saw the latter condition were significantly less likely than those working with the opaque box to begin the task by inserting the tool into the top hole.

These results appear to mirror those obtained by Want and Harris (2001), suggesting that they represent a kind of affordance learning. It certainly does not involve imitation or OMR, for instead, a component of what the model did is explicitly omitted from the subject's task solution. Could we instead have a case of end-state emulation? After all, in a sense, the subject attained the result it saw the model achieve, retrieving food from the front hole, but by a technique that omits earlier parts of what the model did. This would correspond to our nesting of end-state emulation under copying, insofar as subjects copy only the final part of the action sequence they witness. However, we do not favor this account, because we suspect that if the irrelevant part of the task had come in the middle or later, the same tendency to ignore it would have been found. Accordingly, these results seem better interpreted as implying learning about affordancesspecifically, learning that poking the stick into the top hole does not afford any productive causal linkage with achieving the critical outcome of food retrieval.

A study by Bard, Fragaszy, and Visalberghi (1995) represents the alternative approach to identifying affordance learning, outlined earlier-that of investigating whether information acquired can be used in relation to tasks that are different from that observed. Bard et al. showed that if young chimpanzees had mastered the probing of food out of a tube following observation of a model, they were less likely than nonobservers to make errors when faced with more complex problems of this kind that required modification of the tool before it could be used satisfactorily. This finding, however, concerned only two pairs of chimpanzees and, therefore, could not be evaluated statistically; it remains possible that the apparent effects of observation were not affordance learning but merely reflected individual differences. This study is, accordingly, most valuable as a unique demonstration of the potential in this approach.

4. Other categories of social learning. Space does not permit here an equivalent in-depth analysis of the remaining categories of social learning defined in Figure 1, enhancement and observational conditioning; we perforce mention them briefly for completeness. Stimulus enhancement is recognized as a cognitively low-level process, quite widespread among animals (Whiten \& Ham, 1992). As was noted above, when chimpanzees in a twoaction experiment show no evidence of imitation but have clearly learned something nonobserving controls have not (Stoinski \& Whiten, 2003; Stoinski et al., 2001), stimulus enhancement may be involved. However, researchers' preoccupation with the presumably more complex processes of imitation and emulation means that stimulus enhancement is underresearched as a category in its own right. Too often, it is merely the default explanation when more complex processes remain unconfirmed.
Similarly neglected in apes is observational conditioning, a phenomenon principally known from the way macaques have been shown to acquire fear responses to specific objects through observation (Mineka \& Cook, 1988). Although described as social referencing, a term used by those studying human infants, the findings in a single study on this topic by Russell, Bard, and Adamson (1997; see the Appendix) appear to reflect precisely the effect at stake.

\section{ARE APES SPECIAL?}

The above review suggests that apes do indeed ape, in both the narrow and the broad senses. A summary answer to How do apes ape? is that they do so by exploiting a portfolio of quite varied, complementary sociallearning processes at their disposal. Selection within this portfolio will be discussed below in the final section.

However, as was noted earlier, there is now good experimental evidence of imitation in other primates and in birds, and the comparable evidence in apes cited so far may appear to be no stronger (Caldwell \& Whiten, 2002; Heyes, 2001). For example, both starlings and budgerigars have copied the technique of gaining food by either stabbing down a stopper that was in the way or, alternatively, picking it out, according to which model they observed (Campbell, Heyes, \& Goldsmith, 1999; Heyes \& Saggerson, 2002). Likewise, chimpanzees copied either poking bolts out of the way to open an artificial fruit or, alternatively, pulling them out with a twisting action (Whiten et al., 1996). Given that apes have long had a reputation for being special in their social-learning capacities, we might ask how well this reputation is still deserved. Many issues are raised by this question. Here, we will discuss five.

\section{Variety, Novelty, and Manual Dexterity}

A starling can peck or grip things with its bill but can manipulate objects in limited ways, as compared with an ape, who has the use of two, many-jointed, five-fingered hands that offer great flexibility in action repertoires. Each hand is capable of several different grips-on top of which, the hands can be used in complementary ways. The repertoire this may generate is indicated by the catalogue of 72 functionally distinct manipulative actions identified by Byrne, Corp, and Byrne (2001) for mountain gorilla foraging. Adding the use of a variety of tools affords a further combinatorial explosion in the repertoire of potential action patterns and in the complexity of their combinations that might be learnable only by sufficiently sophisticated copying mechanisms.

These contrasts are reflected in the actions that have been shown to be imitated by different species. For example, among birds, the alternatives copied in two-action tests of imitation have included pecking on a treadle versus stepping on it (pigeons, Zentall et al., 1996; quail, Akins \& Zentall, 1998) and stabbing versus picking up a stopper (e.g., starlings, Campbell et al., 1999). The differences between these pairs of alternatives appear to be relatively simple. 
The variety available to apes may be contrasted for both bodily and object-centered patternings of behavior. With respect to bodily imitation, the finding that marmosets would copy mouth versus hand use (Voelkl \& Huber, 2000) and pigeons likewise would copy beak versus foot use (Zentall et al., 1996) was important in classing these as true imitation. Use of different body parts to deal with the same task cannot be explained as emulation but, rather, as imitation that relies on visuomotor mapping from seen parts of the model's body to equivalent parts of the self. In apes, this mapping has been most graphically charted in the do-as-I-do studies. In Custance et al. (1995), for example, coders blind to what each chimpanzee had actually watched identified matching in relation to touching several parts of the body in sight (e.g., the stomach), as well as out of sight (e.g., the back of the head), symmetric and asymmetric conjunctions of hands (e.g., clap back of other hand), digit movements (e.g., wiggle fingers), and head or whole-body actions (e.g., look up, hug self): 34 varieties in all (Custance et al., 1995). The extent of such variation that can be copied at the bodily level appears to far exceed that possible for species that do not possess comparable flexibility. The scope of all that is copied in do-as-I-do studies implies corresponding demands on the underlying action representation systems.

Manipulation of objects and combinations of objects (as in tool use) raises the prospect of additional variety to challenge copying processes. Thus, for example, MyowaYamakoshi and Matsuzawa (1999), having modeled various arbitrary actions on pairs of objects, reported a chimpanzee copying such distinctions as (1) putting a hose into a hole in a stool, (2) hanging the hose around the back of the stool, and (3) pushing the hose against the stool. With respect to functional actions involving objects, Bering, Bjorklund, and Ragan (2000; see the Appendix) reported both orangutans and chimpanzees copying such actions as picking an object up with tongs, which they had not done in baseline trials.

The variations in actions thus engendered have implications for the related issue of novelty. Authors vary in whether they insist on including novelty of the action pattern as a criterion for identifying imitation (Huber, 1998), but many would concur that where there is evidence of novelty, the ascription of imitation is clearer. The generativity in ape behavior outlined above means that the potential for novel action patterns is correspondingly increased. Although no behavior can ever be absolutely novel-just one of the reasons novelty is difficult to measure (Whiten \& Custance, 1996) — the studies cited above to illustrate variety in what apes may imitate all made efforts to establish how improbable the act in question would naturally be for the experimental subjects. Thus, Custance et al. (1995) tested do-as-I-do with a set of actions not used in the training phase and categorized the known novelty of these to the subjects; Myowa-Yamakoshi and Matsuzawa (1999) and Bering et al. (2000) tested for imitation only if a target action had not been spontaneously produced in baseline trials.
Together, these findings suggest that apes may have a capacity for copying a much greater variety of actions that are relatively novel to them that is not shared by many of the other species studied.

Of course, the ape complexities outlined in this section need to be subjected to more direct comparative research (Caldwell \& Whiten, 2002). If they are borne out, this would suggest a functional fit with the variety of putative traditions that studies in the wild suggest are adaptive for apes to acquire (van Schaik et al., 2003; Whiten et al., 1999, 2001). In the case of chimpanzees, for example, the range of such behaviors spans different methods for dispatching ectoparasites (stab with finger, squash on leaf, or inspect on leaf and then eat or discard) and use of a variety of tools, ranging from those for powerful pounding to delicate probing, and grooming techniques (Whiten et al., 2001). It could thus be informative to address further comparisons with species of birds that manipulate tools or other objects in complex ways (Huber, Rechberger, \& Taborsky, 2001; Hunt \& Gray, 2003).

\section{Selective Copying: Sensitivity to Causal \\ Relevance}

Studies with birds have established that social learning can become more likely when the model is seen to gain a reward (pigeons, Palameta \& Lefebvre, 1985; quail, Akins \& Zentall, 1998). This suggests that the imitation identified in Akins and Zentall's study was not mere mimicking (done blindly, irrespective of the end gained) but, instead, involved decision-making processes sensitive to what actions by the model were associated with desirable outcomes.

The experiment by Horner and Whiten (2004), described earlier, took this issue further. This study investigated whether chimpanzees are sensitive to aspects of tool use that, to a human at least, would appear to have no causal connection with desirable outcomes observed. Recall that young chimpanzees faced with the transparent version of the task, in which they could see a sticktool ineffectually beating on a false floor, subsequently tended to omit this action from their efforts. By contrast, when the task was opaque, the action was included significantly more often.

From this, it was concluded that chimpanzees do not merely mimic what they see but perform a cognitive appraisal that identifies plausible causal connections before including an action in any imitation performed (Horner $\&$ Whiten, 2004). This does not necessarily imply a deep grasp of causality but, rather, the appreciation of such rules as that physical connection is necessary for a tool to make desirable outcomes possible. Whether this will turn out to be a phenomenon special to apes we do not know, for we believe that no similar tests have yet been made with other species.

\section{Structural Complexity of Actions}

Byrne (1995) has argued that certain methods of foraging employed by apes are especially structurally com- 
plex and that imitation may occur at a matching level, which he dubbed the program level. Byrne and Russon (1998) defined the latter as "copying the structural organisation of a complex process including the sequence of stages, subroutine structure and bimanual organisation... while furnishing the exact details of actions by individual learning" (p. 676). They offered the foraging techniques of wild gorillas and orangutans' manipulation of human artifacts, such as hammocks, as possible examples, although as the peer discussion accompanying the article emphasized, it was not yet possible to test these hypotheses.

Whiten (1998a) pointed out that imitation might occur in relation to separable parts of Byrne and Russon's (1998) complex definition-most interestingly, with respect to either sequential or hierarchical organization. This surmise was borne out in an experiment designed explicitly to test for copying of these kinds (Whiten, $1999,2002 b$ ), in which young children copied the hierarchical structure of a task, but not the specific sequence of actions models had used to implement it.

With apes, imitation of sequential structure has been tested by allowing chimpanzees to observe one of two alternative models that differed in the sequential order in which they dismantled the defenses on an artificial fruit. Over the course of three trials, incorporating five separate demonstrations, subjects converged (without being rewarded for doing so) almost perfectly on the particular sequencing they had witnessed (Whiten, 1998b).

No such effect was found in replications with gorillas (Stoinski et al., 2001) or orangutans (Stoinski \& Whiten, 2003). However, these negative results should be treated with caution. The two alternative sequential orders of dismantling are, of course, entirely arbitrary, and the absence of copying may reflect not an inability to copy a sequence, but a judgment by the subject that the sequence is irrelevant (young children have also ignored the sequential order of actions, while copying higher level hierarchical structuring; Whiten, 2002c). An alternative kind of experiment is thus one in which the sequential order is, instead, perceivable as logically necessary. This is, in fact, what appeared to be the case in the study by Horner and Whiten (2004) with opaque and transparent boxes, described above. However, before revisiting this, we will describe a recent study in which the copying of hierarchical structure was explicitly addressed, for we can then draw some more integrative conclusions.

The logic of the hierarchically structured artificial fruit (HAF) experiment followed that in experiments performed earlier with children (Whiten, 2002b, 2002c). The HAF used by Marshall-Pescini and Whiten (2004) required 12 different actions to be completed - made up of three sets of the actions remove bolts, peel back lid, slide trap door, and turn knurled wheel - before food became accessible. In the columns method, a model performed each 4-action set (dealing with bolts-lid-doorwheel) as a whole, then proceeded to the next. In the rows method, by contrast, they removed all three bolts in a row, then opened all three doors, then slid all three doors, and finally turned all three wheels. Juvenile chim- panzees showed a significant tendency to copy the hierarchically organized alternative they had seen, the first evidence of imitation of hierarchical structure, we believe, in a nonhuman species. However, it does not seem to conform to Byrne and Russon's (1998) definition of program-level imitation, insofar as the subjects did copy the form of particular aspects of component actions but failed to show copying of sequential order (e.g., left-right, vs. right-left across a row).

We think Horner and Whiten's (2004) study importantly complements these experiments. In the HAF study and in those by Whiten (1998b, 2002b, 2002c), the sequential and hierarchical structures modeled were arbitrary alternatives, whereas in Horner and Whiten's study, they were logically necessary, as they would be naturally. Recall that in the opaque box condition, there was a significant tendency to begin with the top hole and then proceed to the lower one. In the transparent condition, by contrast, the action on the top hole was more likely to be omitted. Thus, we have evidence of sequence copying, manifested systematically in the opaque condition. But there is also copying of what the chimpanzee appears to perceive as hierarchical structure, for the two conditions together show that subjects have a capacity to parse (Byrne, 2000) the component actions, omitting one in the transparent condition, yet assembling both in the observed way in the opaque condition. They thus copied a sequential order that was perceivable as nonarbitrary.

Are apes special in showing these complexities in copying? Chimpanzees are the only animals to have shown these effects, but to our knowledge, other species remain to be tested in comparable ways.

\section{Recognition of the Copying Process}

Whiten (2000) has argued that the very fact that chimpanzees and orangutans have been able to learn the doas-I-do routine means that they have (or can acquire) a concept of imitation. This has been achieved only in great apes (see the Appendix) and dolphins (Herman, 2002). It has not proved possible to train monkeys to perform the task (macaques, Mitchell \& Anderson, 1993; capuchins, Fragaszy, Deputte, Hemery, \& Johnston, unpublished, cited in Visalberghi \& Fragaszy, 2002). It seems unlikely to be a coincidence that great apes (Tomasello \& Call, 1997) and dolphins (Reiss \& Marino, 2001) are also the only species to recognize themselves in mirrors. These taxa appear to share with humans a special capacity for self-representation. In the case of imitation, this constitutes a second-order cognitive process; they recognize the act of imitation itself. The implication of this apparent sophistication for the nature of imitation in apes remains to be understood. One possibility is that it affords a greater sophistication of executive control over when imitation is deployed, in preference to other options distinguished in Figure 1.

\section{Enculturation}

As was noted earlier, the evidence for imitation of the highest fidelity has typically come from apes that have 
been raised with much interaction with humans. Consequently, it has been suggested that their imitativeness results from an enculturation process, in which their attention is channeled in ways like those experienced by children (Tomasello, Kruger, \& Ratner, 1993). Given that this preoccupation is a feature of the ape literature, whereas enculturation is not mentioned by those researching imitation in birds and other taxa, it seems warranted to raise this issue under the heading, Are apes special?

For some, the question of enculturation is a major factor that deserves to be addressed at the start of any review (Tomasello \& Call, 1997). We have not done this for two related reasons. One is a concern that some of the evidence relied on for this distinction has its own problems. Thus, in the foundational study of Tomasello, Savage-Rumbaugh, and Kruger (1993) human-reared and mother-reared chimpanzees were compared, using a task in which the apes were instructed to do what I do. However, the mother-reared subjects were not trained to criterion in the manner of Custance et al. (1995), to check whether they were responding to this cue in the same way as the human-reared subjects. In addition, the latter were, of course, more familiar with their human testers. A trio of studies by Bjorklund and colleagues (see the Appendix), interpreted as supporting the enculturation hypothesis, in fact involved only human-reared subjects.

The second concern is that the enculturation experience may actually be replicating experiences more typical for wild apes, in contrast to those of captive apes that have been deprived of the ontogenetic inputs necessary for the more sophisticated kinds of social learning to develop (Boesch, 1993; Whiten, 1993). Field experiments may be necessary before such matters can be settled. However, it should be noted that the new findings in Horner and Whiten (2004), and Marshall-Pescini and Whiten (2004), described above, were obtained in work with wild-born juveniles living in the Ngamba Island Chimpanzee Sanctuary in Uganda. Just what the necessary developmental inputs to social-learning competence are (Heyes, 2002; Whiten, 2000) and whether apes are at all special in requiring them, as compared with other taxa, are questions that remain to be resolved.

\section{CONCLUSIONS: HOW DO APES APE?}

The evidence that has now accumulated shows that apes do ape, but how they do so cannot be given any simple unitary answer, such as that apes are imitators or that they are emulators. Rather, the picture emerging is that apes may develop a portfolio of social-learning processes that are differentially activated in different contexts and in relation to different functional requirements. We can now begin to sketch the latter in terms of decision trees through which the ape brain works in the course of social learning. Taking the case of learning about a task like the foraging problems documented in the wild and woven into our experiments, we can begin to build a theory of the underlying processes. These processes appear to be enmeshed in hierarchical decision trees, at many points in which the cognitive system of the learner is deciding whether suitable means are already known that will achieve desirable ends observed or, alternatively, whether aspects of the observed means-to-ends need to be adopted. The following sketch of this process is essentially a series of working hypotheses. Here, we will use the shorthand of talking of the learner's making decisions, but of course it is more accurate to say that the brain or some part of the cognitive system is making the decisions, and there is no implication (at least, no necessary one) of conscious decision making.

1. The most basic initial step in the decision tree lies at the level of stimulus or local enhancement, where the learner's attention is drawn to an object or location by another individual but the decision is to tackle the task by using behavioral resources already available to itself, rather than to learn more from the other individual.

2 . Alternatively, the learner may recognize a new outcome or result connected with the actions of the other individual (who now begins, in a minimal way, to deserve the title model) and may recognize that the behavioral resources are at its disposal to recreate this result, rather than utilizing any other information available. This would be end-state emulation. If the learner perceives the outcomes (or in this case, what may be only potential outcomes) as goals, this is goal emulation; if it seeks only to recreate the results it saw, this is result emulation.

3. If, on the other hand, the decision is that the learner does not have the behavioral resources available for emulation, imitation comes into play. However, the imitation process itself incorporates a set of decisions concerned with selective copying. As is indicated in Figure 1 and the studies reviewed earlier, this copying may focus on aspects of either bodily or object actions or, simultaneously, on patterning in both. However, several of the studies reviewed above have suggested that the search process initially focuses on what, in relation to desirable ends, are the closest plausible causal actions of the model. In the case of tool use, this will involve a focus on the actions of the tool itself in relation to its target, and only this aspect of the form of the actions seen may be copied, neglecting the particular bodily movements the model used to make the tool do what it did. The tendency of chimpanzees and orangutans to focus on what manipulated objects are doing, at the expense of bodily fidelity, might reflect this bias.

In suggesting that the learner is identifying plausible causal actions, we are not implying that the learner must have a deep appreciation of the concept of causality. There is much evidence that apes do not closely approach this (Povinelli, Reaux, Theall, \& Giambrone, 2000). What is important is that the learner has some way of functionally recognizing which acts of the model are causing the outcome of interest. This could, in principle, be achieved in a number of ways, such as by identifying strong statistical associations where repeated viewing is possible (Byrne, 2000) or by applying perceptual rules of thumb, such as contingency, contact, and so on, which we alluded to in discussing the study by Horner and Whiten 
(2004), and which could even be used by subjects to learn from one-off demonstrations.

4. Parts of the research reviewed above support Byrne's (1995; Byrne \& Russon, 1998) suggestion that imitation may occur at different levels within an action's organization-for example, at the top levels of overall sequential or hierarchical structure or, alternatively, down at the level of details of certain components. This raises the possibility that apes might have a systematic bias favoring learning at one level rather than at another. Whether this is the case remains unclear as yet, but two different aspects of the question deserve brief discussion.

First, Byrne and Russon (1998) suggested that naturalistic data for gorilla feeding and orangutan object manipulation suggest that the top level (program level) might be favored. However, in the first experimental tests of this hypothesis, evidence of imitation was found primarily in relation to details, and not overall sequencing (gorillas, Stoinski et al., 2001; orangutans, Stoinski \& Whiten, 2003). Stoinski et al. accordingly suggested that we need to entertain the hypothesis converse to that of Byrne and Russon-namely, that these apes might learn critical details by observation and might assemble the overall structure of a complex act themselves, a process Russon (1999) called hierarchization. As Stoinski et al. noted, many of the key findings reviewed by Byrne and Russon, for both wild and tame apes, are quite consistent with this. Clearly, more research is needed to differentiate these alternatives.

A second possibility is that apes initially focus preferentially on one level (high or low) and then, as they master this and build up a scheme of action, focus more social learning on other, complementary levels. Some of our results converge on the conclusion that this may indeed be the case and that details are learned first. Thus, Whiten (1998b) found evidence of copying actions on individual parts of an artificial fruit in the first trials, whereas high fidelity of overall sequential copying emerged only with repeated viewing and performing. Marshall-Pescini and Whiten (2004) found that evidence of hierarchical copying emerged only in older juveniles, whereas imitation of individual elements occurred already in younger infants. Such results suggest that chimpanzees first focus on learning what they perceive to be critical elements of the task and, as they master these, are progressively more able to attend to higher level patterning. However, in the study by Horner and Whiten (2004), evidence of sequential matching was found from the start, together with some imitation of elements closest to the end-result of food being fished out. One explanation for this difference in findings may be that the latter study incorporated a task easier for chimpanzees to parse than the others. Again, more research on this question is clearly needed.

5. A point inherent in the foregoing discussion deserves highlighting in its own right: How apes ape may change in the course of learning a task. Once learning is underway, social learning may become focused on those aspects of the task the learner continues to struggle with. An illustration of this comes from a study by Hirata and
Morimura (2000), which involved chimpanzees probing through a narrow hole for honey. Learners observed those already expert before they made their first attempts, but not after they had been successful. Most interesting, they also observed after making failed attempts at solving the task. Thus, they appeared to selectively attend to a model in order to gain the specific information they needed.

Conversely, there is some limited evidence for conformity effects, whereby the learner, having achieved some competence, converges on the actions of others even though it has used other approaches with success (Whiten, 1998b).

6. Instead of trying to copy the model, the learner may favor learning the affordances of tools or other objects involved and may use this knowledge (learning that, rather than learning how) to construct its own attempt at the task. Such a process may often be difficult to discriminate from imitation, but as we discussed earlier, it may be manifested in selective copying that omits elements that do not have productive affordances or in transfer of learning to other kinds of tasks.

In conclusion, we believe that the emerging answer to how apes ape is, accordingly, not a unitary one but, instead, is best expressed in terms of biases and choices among the varied range of options we have summarized above. Some of these reflect inherent limitations, but others represent adaptive flexibility. The last 15 years have yielded a rich cluster of new insights into ape social learning, and along the way, the research involved has highlighted theoretical and methodological implications important for the wider field of animal social learning.

\section{REFERENCES}

AkIns, C. K., \& Zentall, T. R. (1998). Imitation in Japanese quail: The role of reinforcement of demonstrator responding. Psychonomic Bulletin \& Review, 5, 694-697.

Bard, K. A., Fragaszy, D., \& Visalberghi, E. (1995). Acquisition and comprehension of a tool-using behavior by young chimpanzees (Pan troglodytes): Effects of age and modeling. International Journal of Comparative Psychology, 8, 47-68.

Bering, J. M., BJoRKLund, D. F., \& RAGAN, P. (2000). Deferred imitation of object-related actions in human-reared juvenile chimpanzees and orangutans. Developmental Psychobiology, 36, 218-232.

BJoRklund, D. F., BERING, J. M., \& RAGAN, P. (2000). A two-year longitudinal study of deferred imitation of object manipulation in a juvenile chimpanzee (Pan troglodytes) and orangutan (Pongo pygmaeus). Developmental Psychobiology, 37, 229-237.

BJorklund, D. F., Yunger, J. L., Bering, J. M., \& RAGAN, P. (2002). The generalization of deferred imitation in enculturated chimpanzees (Pan troglodytes). Animal Cognition, 5, 49-58.

BOESCH, C. (1993). Towards a new image of culture in wild chimpanzees? Behavioral \& Brain Sciences, 16, 514-515.

BYRNE, R. W. (1995). The thinking ape: Evolutionary origins of intelligence. New York: Oxford University Press.

BYRNe, R. W. (1998). Commentary on Boesch, C. and Tomasello, M. Chimpanzee and human culture. Current Anthropology, 39, 604-605.

BYRNE, R. W. (2000). Imitation without intentionality: Using stringparsing to copy the organization of behaviour. Animal Cognition, 2, $63-72$.

Byrne, R. W., Corp, N., \& Byrne, J. M. E. (2001). Estimating the complexity of animal behaviour: How mountain gorillas eat thistles. Behaviour, 138, 525-557.

BYRNE, R. W., \& Russon, A. E. (1998). Learning by imitation: A hierarchical approach. Behavioral \& Brain Sciences, 21, 667-721. 
CAldwell, C. A., \& Whiten, A. (2002). Evolutionary perspectives on imitation: Is a comparative psychology of social learning possible? Animal Cognition, 5, 193-208.

CALl, J. (2001). Body imitation in an enculturated orangutan (Pongo pygmaeus). Cybernetics \& Systems, 32, 97-119.

CALL, J., \& CARPENTER, M. (2002). Three sources of information in social learning. In K. Dautenhahn \& C. L. Nehaniv (Eds.), Imitation in animals and artifacts (pp. 211-228). Cambridge, MA: MIT Press.

CAll, J., \& Tomasello, M. (1994). The social learning of tool use by orangutans. Human Evolution, 9, 297-313.

CAll, J., \& Tomasello, M. (1995). The use of social information in the problem-solving of orangutans (Pongo pygmaeus) and human children (Homo sapiens). Journal of Comparative Psychology, 109, 308-320.

Campbell, F. M., Heyes, C. M., \& Goldsmith, A. R. (1999). Stimulus learning and response learning by observation in the European starling, in a two-object/two-action test. Animal Behaviour, 58, 151-158.

Celli, L. M., Tomonaga, M., Udono, T., Teramoto, M., \& Nagano, K. (2001). Learning processes in the acquisition of a tool using task by captive chimpanzees. Psychologia, 44, 70-81.

Custance, D. M. (1998). Apes ape! Behavioral \& Brain Sciences, 21 118-119.

Custance, D. M., Whiten, A., \& Bard, K. A. (1995). Can young chimpanzees (Pan troglodytes) imitate arbitrary actions? Hayes and Hayes (1952) revisited. Behaviour, 132, 837-859

Custance, D. M., Whiten, A., \& Fredman, T. (1999). Social learning of artificial fruit processing in capuchin monkeys (Cebus apella). Journal of Comparative Psychology, 113, 13-23.

Custance, D. M., Whiten, A., SAmbrook, T., \& Galdikas, B. (2001) Testing for social learning in the 'artificial fruit' processing of wildborn orangutans (Pongo pygmaeus), Tanjung Puting, Indonesia. Animal Cognition, 4, 305-313.

Dautenhahn, K., \& Nehaniv, C. L. (EDS.) (2002). Imitation in animals and artifacts. Cambridge, MA: MIT Press.

GALEF, B. G., JR. (1988). Imitation in animals: History, definition, and interpretation of data from the psychological laboratory. In T. R. Zentall \& B. G. Galef, Jr. (Eds.), Social learning: Psychological and biological perspectives (pp. 3-28). Hillsdale, NJ: Erlbaum.

GALEF, B. G., JR. (1990). Traditions in animals: Field observations and laboratory analyses. In M. Bekoff \& D. Jamieson (Eds.), Interpretation and explanation in the study of animal behaviour (pp. 74-95). Boulder: Westview Press.

GALEF, B. G., JR. (1992). The question of animal culture. Human Nature, 3, 157-178.

GALEF, B. G., JR. (2004). Approaches to the study of traditional behaviors of free-living animals. Learning \& Cognition, 32, 53-61.

GALEF, B. G., JR. (in press). Breathing new life into the study of animal imitation: What and when do chimpanzees imitate? In S. Hurley \& N. Chater (Eds.), Perspectives on imitation: From cognitive neuroscience to social science. Boston, MA: MIT Press.

GoODALL, J. (1968). The behaviour of free-living chimpanzees in the Gombe Stream Reserve. Animal Behaviour Monographs, 1, 161-311.

GoodAlL, J. (1973). Cultural elements in a chimpanzee community. In E. W. Menzel (Ed.), Precultural primate behaviour (Vol. 1, pp. 144 184). Basel: Karger.

Hannah, A., \& MCGREw, W. (1987). Chimpanzees using stones to crack open oil palm nuts in Liberia. Primates, 28, 31-46.

HAYES, C. (1951). The ape in our house. New York: Harper.

HAYES, K. J., \& HAYES, C. (1952). Imitation in a home-raised chimpanzee. Journal of Comparative \& Physiological Psychology, 45 450-459.

Herman, L. M. (2002). Vocal, social and self-imitation by bottlenosed dolphins. In K. Dautenhahn \& C. L. Nehaniv (Eds.), Imitation in animals and artifacts (pp. 63-108). Cambridge, MA: MIT Press.

Heyes, C. M. (1993). Imitation, culture and cognition. Animal Behaviour, 46, 999-1010.

Heyes, C. M. (2001). Causes and consequences of imitation. Trends in Cognitive Sciences, 5, 253-261.

HEYES, C. M. (2002). Transformational and associative theories of imitation. In K. Dautenhahn \& C. L. Nehaniv (Eds.), Imitation in animals and artifacts (pp. 501-523). Cambridge, MA: MIT Press.

Heyes, C. M., Jaldow, E., Nokes, T., \& Dawson, G. R. (1994). Imita- tion in rats: The role of demonstrator action. Behavioral Processes, 32, 173-182.

Heyes, C. M., \& SAggerson, A. (2002). Testing for imitative and nonimitative social learning in the budgerigar using a two-object/twoaction test. Animal Behaviour, 64, 851-859.

Hirata, S., \& Morimura, N. (2000). Naive chimpanzees' (Pan troglodytes) observation of experienced conspecifics in a tool-using task. Journal of Comparative Psychology, 114, 291-296.

Horner, V., \& Whiten, A. (2004). Causal knowledge and imitation/ emulation switching in chimpanzees (Pan troglodytes) and children. Manuscript under review.

Huang, C.-T., Heyes, C., \& Charman, T. (2002). Infants' behavioral reenactment of "failed attempts": Exploring the roles of emulation learning, stimulus enhancement, and understanding of intentions. Developmental Psychology, 38, 840-855.

HubER, L. (1998). Movement imitation as faithful copying in the absence of insight. Behavioural \& Brain Sciences, 21, 694.

Huber, L., Rechberger, S., \& TABorsky, M. (2001). Social learning affects object exploration and manipulation in keas, Nestor notabilis. Animal Behaviour, 62, 945-954.

Hunt, G. R., \& GRAY, R. D. (2003). Diversification and cumulative evolution in New Caledonian crow tool manufacture. Proceedings of the Royal Society of London: Series B, 270, 867-874.

Hurley, S., \& ChATER, N. (EDS.) (in press). Perspectives on imitation: From cognitive neuroscience to social science. Cambridge, MA: MIT Press.

IMANISHI, K. (1957). Identification: A process of enculturation in the subhuman society of Macaca fuscata. Primates, 1, 1-29.

Laland, K., Odling-SmeE, J., \& Feldman, M. W. (2000). Niche construction, biological evolution and cultural change. Behavioral \& Brain Sciences, 23, 131-175.

Lloyd-Morgan, C. L. (1896). Habit and instinct. London: Edward Arnold.

MacFarland, D. J. (1984). Animal behaviour. London: Pitman.

MARSHALl-PESCINI, S., \& Whiten, A. (2004). Sociallearning of a hierarchically organised artificial foraging task by infant and juvenile chimpanzees. Manuscript under review.

MCGREW, W. C. (1992). Chimpanzee material culture: Implications for human evolution. Cambridge: Cambridge University Press.

Meltzoff, A. N. (1995). Understanding the intentions of others: Reenactment of intended acts by 18-month-old children. Developmental Psychology, 31, 838-850.

Meltzoff, A. N., \& Prinz, W. (2002). The imitative mind: Development, evolution and brain bases. Cambridge: Cambridge University Press.

Miklosi, A. (1999). The ethological analysis of imitation. Biological Review, 74, 347-374.

Miles, H. L., Mitche Ll, R. W., \& Harper, S. E. (1996). Simon says: The development of imitation in an enculturated orangutan. In A. Russon, K. A. Bard, \& S. T. Parker (Eds.), Reaching into thought: The minds of the great apes (pp. 278-299). Cambridge: Cambridge University Press.

MiNEKA, S., \& COOK, M. (1988). Social learning and the acquisition of snake fear in monkeys. In T. R. Zentall \& B. J. Galef, Jr. (Eds.), Social learning: Psychological and biological perspectives (pp. 51-73). Hillsdale, NJ: Erlbaum.

MitCHe LL, R. W. (1999). Scientific and popular conceptions of the psychology of great apes from the 1790s to the 1970s: Déjà vu all over again. Primate Report, 53, 1-118.

Mitchell, R. W., \& Anderson, J. R. (1993). Discrimination learning of scratching, but failure to obtain imitation and self-recognition in a long-tailed macaque. Primates, 34, 301-309.

Myowa, M. (1996). Imitation of facial gestures by an infant chimpanzee. Primates, 37, 207-213.

MYOWA-YAMAKOSHI, M., \& MATSUZAWA, T. (1999). Factors influencing imitation of manipulatory actions in chimpanzees (Pan troglodytes). Journal of Comparative Psychology, 113, 128-136.

Myowa-Yamakoshi, M., \& MatsuzaWA, T. (2000). Imitation of intentional manipulatory actions in chimpanzees (Pan troglodytes). Journal of Comparative Psychology, 114, 381-391.

Nagell, K., Olguin, R. S., \& Tomasello, M. (1993). Processes of social learning in the tool use of chimpanzees (Pan troglodytes) and human children (Homo sapiens). Journal of Comparative Psychology, 107, 174-186. 
NisHidA, T. (1987). Local traditions and cultural transmission. In B. B Smuts, D. L. Cheney, R. M. Seyfarth, R. W. Wrangham, \& T. T. Struhsaker (Eds.), Primate societies (pp. 462-474). Chicago: University of Chicago Press.

Palameta, B., \& Lefebvre, L. (1985). The social transmission of a food finding technique in pigeons: What is learned? Animal Behaviour, 33, 892-896.

Paquette, D. (1992). Discovering and learning tool-use for fishing honey by captive chimpanzees. Human Evolution, 7, 17-30.

Passingham, R. E. (1982). The human primate. New York: Freeman.

Povinelli, D. J., ReauX, J. E., Theall, L. A., \& Giambrone, S. (2000). The question of tool modification. In D. J. Povinelli (Ed.), Folk physics for apes: A chimpanzee's theory of how the world works (pp. 271-296). Oxford: Oxford University Press.

REISS, D., \& MARINO,L. (2001). Mirror self-recognition in the bottlenose dolphin: A case of cognitive convergence. PNAS, 98, 5937-5942

Rizzolatti, G., Fadiga, L., Fogassi, L., \& Gallese, V. (2002). From mirror neurons to imitation: Facts and speculations. In A. N. Meltzoff \& W. Prinz (Eds.), The imitative mind (pp. 247-266). Cambridge: Cambridge University Press.

Russell, C. L., BARd, K. A., \& AdAmson, L. B. (1997). Social referencing by young chimpanzees (Pan troglodytes). Journal of Comparative Psychology, 111, 185-193.

Russon, A. E. (1999). Orangutans' imitation of tool use: A cognitive interpretation. In S. T. Parker, R. W. Mitchell, \& H. L. Miles (Eds.), Mentalities of gorillas and orangutans (pp. 119-145). Cambridge: Cambridge University Press.

SHETTLEWORTH, S. J. (1998). Cognition, evolution and behaviour. Oxford: Oxford University Press.

Stoinski, T. S., \& Whiten, A. (2003). Social learning by orangutans (Pongo pygmaeus) in a simulated food processing task. Journal of Comparative Psychology, 117, 272-282.

Stoinski, T. S., Wrate, J. L., URe, N., \& Whiten, A. (2001). Imitative learning by captive western lowland gorillas (Gorilla gorilla gorilla) in a simulated food-processing task. Journal of Comparative Psychology, 115, 272-281.

TOMASELLO, M. (1990). Cultural transmission in the tool use and communicatory signalling of chimpanzees? In S. Parker \& K. Gibson (Eds.), Language and intelligence in monkeys and apes: Comparative developmental perspectives (pp. 274-311). Cambridge: Cambridge University Press.

Tomase Llo, M. (1996). Do apes ape? In C. M. Heyes \& B. G. Galef, Jr. (Eds.), Social learning in animals: The roots of culture (pp. 319-346). London: Academic Press.

ToMASELlo, M. (1998). Emulation learning and cultural learning. Behavioral \& Brain Sciences, 21, 703-704.

Tomasello, M., \& CAll, J. (1997). Primate cognition. New York: Oxford University Press.

Tomase llo, M., Call, J., \& Gluckman, A. (1997). Comprehension of novel communicative signs by apes and human children. Child Development, 68, 1067-1080.

Tomasello, M., DAVIS-DASIlva, M., CAMAK, L., \& BARD, K. (1987). Observational learning of tool-use by young chimpanzees. Human Evolution, 2, 175-183.

Tomasello, M., Kruger, A. C., \& Ratner, H. H. (1993). Cultural learning. Behavioral \& Brain Sciences, 16, 495-552.

Tomasello, M., Savage-Rumbaugh, S., \& Kruger, A. C. (1993). Imitative learning of actions on objects by children, chimpanzees, and enculturated chimpanzees. Child Development, 64, 1688-1705.

TONOOKA, R., TOMONAGA, M., \& MATSUZAWA, T. (1997). Acquisition and transmission of tool making and use for drinking juice in a group of captive chimpanzees. Japanese Psychological Research, 39, 253-265.

Toth, N., Schick, K. D., Savage-Rumbaugh,E. S., Sevcik, R. A., \& RUMBAUGH, D. M. (1993). Pan the tool-maker: Investigations into the stone tool-making and tool-using capabilities of a bonobo (Pan paniscus). Journal of Archaelogical Science, 20, 81-91.

VAN SChaik, C. P., ANCRENAZ, M., Borgen, G., GALDIKAs, B., KNOTt, C. D., Singleton, I., SuZuki, A., UtAMI, S. S., \& Merrill, M. (2003). Orangutan cultures and the evolution of material culture. Science, 299, 102-105.
Visalberghi,E., \& Fragaszy, D. (1990). Do monkeys ape? In S. Parker \& K. Gibson (Eds.), Language and intelligence in monkeys and apes: Comparative developmental perspectives (pp. 247-273). Cambridge: Cambridge University Press.

VisALBERGHI,E., \& FrAGASZY, D. M. (2002). Do monkeys ape? Ten years after. In K. Dautenhahn \& C. L. Nehaniv (Eds.), Imitation in animals and artifacts (pp. 471-500). Cambridge, MA: MIT Press.

VoelkL, B., \& Huber, L. (2000). True imitation in marmosets. Animal Behaviour, 60, 195-202.

WANT, S. C., \& HARRIS, P. L. (2001). Learning from other people's mistakes: Causal understanding in learning to use a tool. Child Development, 72, 431-443.

WANT, S. C., \& HARRIS, P. L. (2002). How do children ape? Applying concepts from the study of non-human primates to the developmental study of "imitation" in children. Developmental Science, 5, 1-13.

WhiteN, A. (1993). Human enculturation, chimpanzee enculturation and the nature of imitation: Commentary on Cultural learning, by M. Tomasello et al. Behavioral \& Brain Sciences, 16, 538-539.

Whiten, A. (1998a). How imitators represent the imitated: The vital experiments. Behavioral \& Brain Sciences, 21, 707-708.

Whiten, A. (1998b). Imitation of the sequential structure of actions by chimpanzees (Pan troglodytes). Journal of Comparative Psychology, 112, 270-281.

WHITEN, A. (1999). Imitation of sequential and hierarchical structure in action: Experimental studies with children and chimpanzees. In Proceedings of the Artificial Intelligence and Simulation of Behaviour Convention (AISB): Symposium on Imitation in Animals and Artifacts (pp. 38-46). Edinburgh: Society for the Study of Artificial Intelligence and Simulation of Behaviour.

Whiten, A. (2000). Primate culture and social learning. Cognitive Science, 24, 477-508.

Whiten, A. (2002a). From the field to the laboratory and back again: Culture and "social mind" in primates. In M. Bekoff, C. Allen, \& G. M. Burghardt (Eds.), The cognitive animal (pp. 385-392). Cambridge, MA: MIT Press.

WHITEN, A. (2002b). Imitation of sequential and hierarchical structure in action: Experimental studies with children and chimpanzees. In K. Dautenhahn \& C. L. Nehaniv (Eds.), Imitation in animals and artifacts (pp. 191-209). Cambridge, MA: MIT Press.

Whiten, A. (2002c). The imitator's representations of the imitated. In A. Meltzoff \& W. Prinz (Eds.), The imitative mind (pp. 98-121). Cambridge: Cambridge University Press.

Whiten, A., \& Custance, D. M. (1996). Studies of imitation in chimpanzees and children. In C. M. Heyes \& B. G. Galef, Jr. (Eds.), Social learning in animals: Roots of culture (pp. 291-318). London: Academic Press.

Whiten, A., Custance, D. M., Gomez, J.-C., Teixidor, P., \& Bard, K. A. (1996). Imitative learning of artificial fruit processing in children (Homo sapiens) and chimpanzees (Pan troglodytes). Journal of Comparative Psychology, 110, 3-14.

Whiten, A., Goodall, J., MCGrew, W. C., Nishida, T., Re Ynolds, V., Sugiyama, Y., Tutin, C. E. G., Wrangham, R. W., \& Boesch, C. (1999). Cultures in chimpanzees. Nature, 399, 682-685.

Whiten, A., Goodall, J., McGrew, W. C., Nishida, T., Reynolds, V., Sugiyama, Y., Tutin, C. E. G., Wrangham, R. W., \& Boesch, C. (2001). Charting cultural variation in chimpanzees. Behaviour, 138, 1481-1516.

WHITEN, A., \& HAM, R. (1992). On the nature and evolution of imitation in the animal kingdom: Reappraisal of a century of research. In P. J. B. Slater, J. S. Rosenblatt, C. Beer, \& M. Milinski (Eds.), Advances in the study of behavior (pp. 239-283). New York: Academic Press.

Whiten, A., Horne R, V., \& Marshall-Pescini, S. R. J. (2003). Cultural panthropology. Evolutionary Anthropology, 12, 92-105.

Wood, D. (1989). Social interaction as tutoring. In M. H. Bornstein \& J. S. Bruner (Eds.), Interaction in human development (pp. 59-80). Hillsdale, NJ: Erlbaum.

Zentall, T. R. (2004). Action imitation in birds. Learning \& Cognition, 32, 15-23.

Zentall, T. R., Sutton, J. E., \& Sherburne, L. M. (1996). True imitative learning in pigeons. Psychological Science, 7, 343-346. 


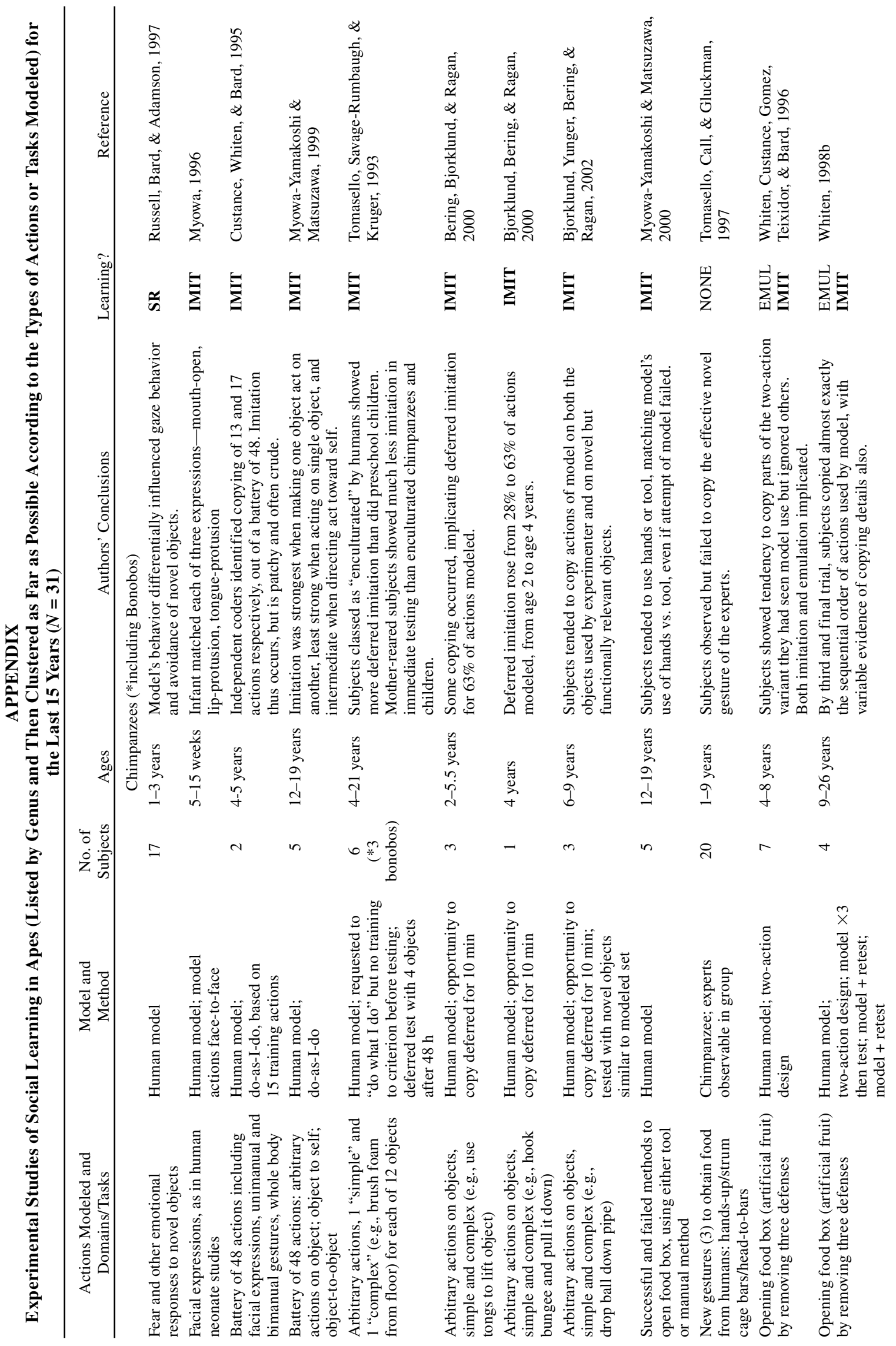




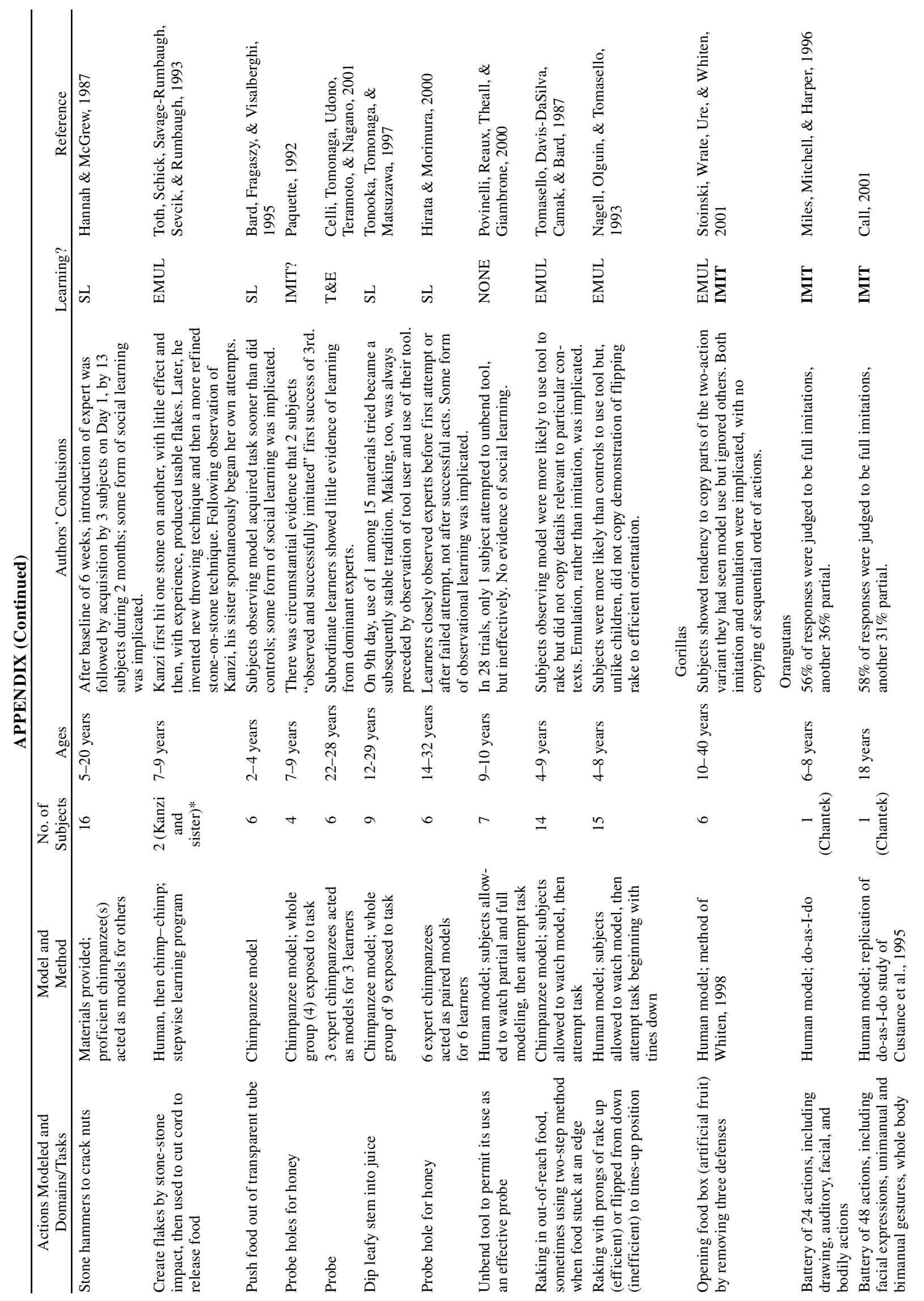




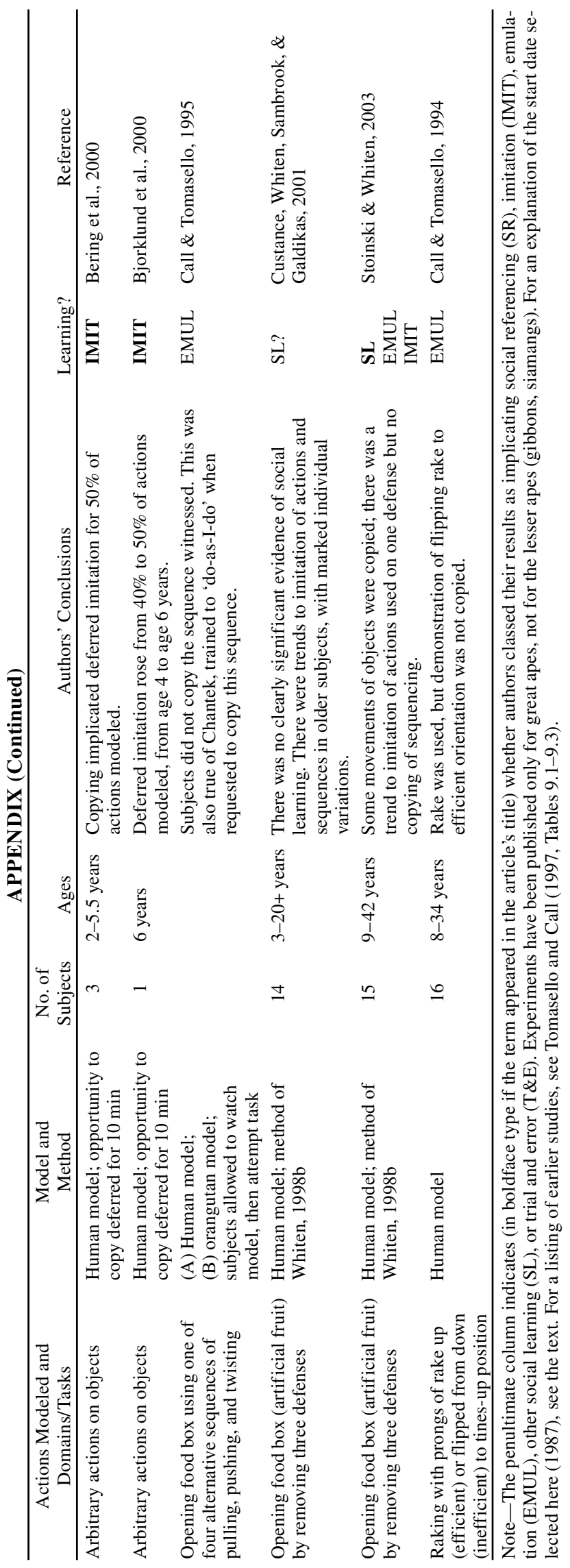

